\title{
Analysis of Unidirectional Aligned Banana and Glass Fibre Reinforced Polyester Composite for Tensile and Flexural Strength
}

\author{
Vatsal Maisuriya $^{1}$, Piyush Jain ${ }^{2, *}$, Hitesh Jariwala ${ }^{3}$, Damyanti Badagha ${ }^{4}$ \\ ${ }^{1}$ Research Scholar, S. N. Patel Institute of Technology \& Research Centre, Umrakh, Gujarat \\ Technological University, Gujarat, India \\ ${ }^{2}$ Professor, Mechanical Engineering, S. N. Patel Institute of Technology \& Research Centre, Umrakh, \\ Gujarat Technological University, Gujarat, India \\ ${ }^{3}$ Head, Mechanical Engineering, Jayvantrai Harrai Desai Polytechnic, Palsana, Gujarat Technological \\ University Gujarat, India \\ ${ }^{4}$ Assistant Professor, Civil Engineering, S. N. Patel Institute of Technology \& Research Centre, \\ Umrakh, Gujarat Technological University, Gujarat, India
}

\begin{abstract}
The hybrid composite can bedeveloped by utilizing at leasttwo fibrereinforcement in the composite. In the present work, the hybrid composite wasmade by utilizing bananalglass fibrekeeping fibrevolume loading 40\%. The banana fibre was treated with $5 \% \mathrm{NaOH}$. The tensile test and flexural test were preceded according to ASTM D3039 and ASTM D790respectively.The results indicate that hybrid composite made with $5 \% \mathrm{NaOH}$ treated banana fibre and glass fibre shows improved mechanical properties than non-treated fibre composite.
\end{abstract}

Keywords: Banana fibre; E-glass fibre; Flexural properties; Tensile properties; Unsaturated Polyester resin

*Author for Correspondence E-mail: JAIN_PIYUSH@gtu.edu.in

\section{INTRODUCTION}

The fibre reinforced polymer (FRP) compositematerial has been utilized in various applications as a customary development material for light load application. The high density to weight proportion, corrosion resistant property and low support cost are the appealing properties of FRP materials. This advantage of FRP material encourages the researchers to do more innovations. Composite materials are designed and developed using at least two constituent materials with fundamentally unique physical properties which stay independent and particular inside the composite structure. Most of the composites have high strength and more weight due to high density of reinforced fibrebonded in the matrix. So, the goal is generally to make a composite material which is strong and durable, regularly with a low density and weight. These days, composite material generally utilized as substitution of ordinary plastic. Ordinarily thermosetting were used if it getsreinforced, at that point its properties get improved, by the idea of reinforcement of the fibre in the composite. The blend of at least two fibre in reinforcement is characterized as hybridization. The hybrid improves mechanical properties by giving the benefits of two different reinforced fibre characteristics. The portion of the synthetic fibre based hybrid composite have dominating influenced factors like variety in volume/weight division in stacking progression of fibre layer in FRP [1]. While incorporation of natural fibre was likewise revealed as an appropriate swapbecause of its high elongation which makes it as a potential substitute forautomobilesaslight to medium loadapplication [2]. The alkaline treated banana fibre gives improved mechanical 
properties and less water absorption [3]. $\mathrm{NaOH}$ treatment was found to improve mechanical properties because of expelled of waxy layers from the surface of the fibre, In alkaline $5 \%$ of $\mathrm{NaOH}$ concentration preferred best over the other $1 \%, 3 \%, 7 \%, 9 \%$ of concentration[4].Until now the researchers have studied that the convectional composite replaced by adding the Natural fibre for light load application. Researchers found that mono fibre composite achieves lower strength than hybrid composite with same volume fraction, synthetic fibre with natural fibre as a reinforcement in matrix gives better adhesion composite structure improved mechanical properties in hybridization, when testing done as per ASTM standard. For hybrid composite most of the work reported on combination of glass fibre and carbon fibre with epoxy as a matrix, but both fibre have higher density, realistic advantage of hybrid is not fulfilled so, more experimental investigation is required using the combination of glass and natural fibre with polyester resin. In the experimental work, deficiency observed with combination of glass and natural fibre as well as natural with natural fibre with thermosetting binders. Moreover, to analyze mechanical properties like tensile strength and 3 point bending(flexure) test with treated and untreated fibre of long continuous unidirectional aligned (natural + synthetic) fibre reinforced plastics, more work needs to be done. Hybridize with treated fibre also needs to be investigated for different natural fibre based on volume fraction and test as per ASTM standard. In the recent experimental work, the mechanical properties of composite with $40 \%$ of fibre loading were studied. The point of this examination is to research the tensile properties in hybrid fibre reinforced composite by hand layup technique. To explore the conduct of such unique reinforcement in $40 \%$ of volume fraction of fibre according to ASTMD 3039 and ASTMD 790 tests followed by the experimental and analytical investigation done with the comparison. The objective of this work is to give a thorough comprehension about the impact of fibre hybridization in improving the mechanical properties of composite. The specific properties of the natural fibre composites were found better than those of glass fibre. This recommended that natural fibre composites have a potential to replace glass in many engineering applications which do not require very high load bearing capabilities [16]. Generally, hybrid composite made via carbon fibre and glass, having high density, thickness and weight increment so natural fibre having less thickness can be use as reinforced with glass fibre give great outcome for light load application in automobile[5].

\section{RULE OF MIXTURE}

There are two unique approaches to choose the mechanical properties of hybrid composite materials, which are either using the weight fraction or using the volume fraction for fabrication of the composite. The strength and modulus of the hybrid composite can be derived from the Rule of hybrid mixture equation by neglecting the interaction between two system [6]. Rule of mixture and Rule of hybrid mixture were resolved subjected to the volume fraction of the composite constituents. Rule of mixture approach can be used to decide the vast majority of composite lamina properties[15].

Its equation as $\left(\mathrm{W}_{\mathrm{c}}=\mathrm{W}_{\mathrm{f}}+\mathrm{W}_{\mathrm{m}}\right),\left(\mathrm{V}_{\mathrm{c}}=\mathrm{V}_{\mathrm{f}}+\right.$ $\mathrm{V}_{\mathrm{m}}$ ).

$$
E_{c}=E_{f 1} v_{f 1}+E_{f 2} v_{f 2}+E_{m} v_{m}
$$

Theoretical calculation of tensile strength $\sigma_{\mathrm{c}}=\sigma_{\mathrm{f} 1} \mathrm{~V}_{\mathrm{f} 1}+\sigma_{\mathrm{f} 2} \mathrm{~V}_{\mathrm{f} 2}+\sigma_{\mathrm{m}}\left(1-\mathrm{V}_{\mathrm{f} 1}-\right.$ $\mathrm{Vf} 2$ (3)

Theoretical calculation of flexure strength by equation $\sigma=3 F L / 2 B H 2$

[from ASTMD 790]

\section{EXPERIMENTAL WORK}

1. Materials

In this experiment, Glass and banana fibre is used for specimen preparation. Thermosetting resin is used as a matrix material that is polyester resin for the fabrication of hybrid composite. The glass fibre utilized in the current work is E-glass fibre which was provided by Triveni Fibre, Surat, Gujarat, India. The unsaturated polyester resin bought 
from Sundaram Chemicals, utilized in the present work along with methyl ethyl ketone peroxide (hardener) and cobalt napthane (quickening agent) in ratio of 1:100in resin Composition of glass fibre:

Various types of glass fibre are manufactured based on the compositions. In all of the glass fibre Silica and calcium oxide play major role. The other constituents used in glass fibre are given in the Table 1 below.[7]

Table 1: Composition of glass fibre [7].

\begin{tabular}{|l|l|l|l|}
\hline Alloy (\%) & E-glass & C-glass & S-glass \\
\hline $\mathrm{SiO}_{2}$ & 55.2 & 65.0 & 65.0 \\
\hline $\mathrm{Al}_{2} \mathrm{O}_{3}$ & 8.0 & 4.0 & 25.0 \\
\hline $\mathrm{CaO}$ & 18.7 & 14.0 & - \\
\hline $\mathrm{MgO}$ & 4.6 & 3.0 & 10.0 \\
\hline $\mathrm{Na}_{2} \mathrm{O}$ & 0.3 & 8.5 & 0.3 \\
\hline $\mathrm{K}_{2} \mathrm{O}$ & 0.2 & - & - \\
\hline $\mathrm{B}_{2} \mathrm{O}$ & 7.3 & 5.0 & - \\
\hline
\end{tabular}

Glass fibre were obtained from sunduram chemicals Surat, Gujarat. Addition of glass fibre improves the mechanical strength and reduces the wear. The composites specimens fabricated by using hand layup method. Mechanical properties of tested glass fibre are given Table 2.

Table 2: Mechanical properties of glass fibre.

\begin{tabular}{|c|c|}
\hline Properties & E-glass fibre \\
\hline Density $\mathrm{g} / \mathrm{cm}^{3}$ & 2.5 \\
\hline Tensile Strength MPa & 1700 \\
\hline Modulus of Elasticity GPa & 72 \\
\hline Flexure strength Mpa & 245 \\
\hline Elongation \% & 3.8 \\
\hline Flexure modulus GPa & 1.52 \\
\hline
\end{tabular}

a. Properties of banana fibre:

Banana fibre were obtained from Agriculture University Navsari, Gujarat and were extracted using rasporder machine. Banana fibre are obtained from waste products of banana cultivation. Because of high cellulose content, it shows good tensile properties. Mechanical properties of tested banana Fibre are given in the following Table 3.

Table 3: Mechanical properties of banana fibre.

\begin{tabular}{|l|l|}
\hline Properties & Value \\
\hline Density $\mathrm{g} / \mathrm{cm}^{3}$ & 1.35 \\
\hline Elongation at break \% & 5.9 \\
\hline Tensile Strength MPa & 340 \\
\hline Diameter $\mu \mathrm{m}$ (micron) & 1387 \\
\hline Flexure strength Mpa & 2.5 \\
\hline Young's modulus GPa & 27 \\
\hline
\end{tabular}

\section{b. Polyester Resin}

This material can be strong yet it relies upon the speed of the response by which it happens. Along with unsaturated polyester resin, $1 \%$ of cobalt quickening agent and $1 \%(\mathrm{vol} \%)$ of MEKP as hardener is utilized. Polyester resin and supporting agent respond together to shape a matrix for the composite. Suitable amount of impetus is significant as it helps the best possible fix of polyester resin framework.Typical Properties of tested Unsaturated Polyester resin are shown in Table 4.

Table 4: Typical Properties of tested Unsaturated Polyester Resin.

\begin{tabular}{|c|c|}
\hline Properties & Polyester resin \\
\hline Density $\mathrm{g} / \mathrm{cm}^{3}$ & 1.2 \\
\hline Tensile Strength MPa & 38.81 \\
\hline Modulus of Elasticity MPa & 1312 \\
\hline Flexure strength Mpa & 44.21 \\
\hline Elongation \% & 4.673 \\
\hline Max. Extension mm & 6.91 \\
\hline Flexure modulus GPa & 1.52 \\
\hline
\end{tabular}

c. Surface modification of fibre

Here, banana fibre treated with alkaline treatment to improve surface modification of the fibre which results for better fibre and matrix bonding according to researcher study $[4,8-11]$. For this concentration is taken as $5 \%$ by weight in distilled water arrangement. It is proved that alkali treatment achieve higher strength [12]. In treatment of the fibre, first wash fibre with fresh water to expel dust, then permit drying it under sunlight for $48 \mathrm{hrs}$. After that all fibre splashed with $5 \% \mathrm{NaOH}$ arrangement. Take 1 liters of distilled water and 50gram of $\mathrm{NaOH}$ pellets at room temperature and add pellets in distilled water. After this, it was blended precisely so that $\mathrm{NaOH}$ blend gets broke up and dissolved in distilled water. Banana fibrelater on added into the arrangement and treated with the arrangement with 6 to 8 hour and again washed with distilled water to neutralize the fibre.

2. Preparation of composite specimen To prepare the composite specimen, the mould developed from the prepared teak wood of size $300 \mathrm{~mm} \times 170 \mathrm{~mm} \times 3 \mathrm{~mm}$. The planning of composite was completed utilizing hand lay- 
up technique. Top cover plate and base surface covered with gel coat for simple evacuation of lamina plate. Fibre were cut according to required measurement and organized unidirectionally in the die with firmly fitted by clamp or fasteners. The fibre volume part of the composite was fixed to forty percent. Right now resin along with hardener and quickening agent were blended in with proportion of 100:1 which is utilized as grid. The air bubble was evacuated cautiously with a roller. After 24 hours composite plate taken out for marking and cutting according to ASTM standard.

3. Mechanical Testing of composite specimen A. Tensile test

The tensile strength of the composite specimen was experimentally evaluated according to ASTM D3039 standard on Universal Testing Machine (UTM) made by Tinius Olsen/larrangement H50kL. The components of the test parameters like measured length and cross head speed were chosen according to ASTM D3039 standard. The specimen was mounted between two jaws of the UTM and tensile test was performed. The dimensions of the specimen are $3 \mathrm{~mm} \times 15 \mathrm{~mm}$ x $250 \mathrm{~mm}$ where $50 \mathrm{~mm}$ at both the ends is kept for tab. The specimens for tensile testing are shown in Figure. 1 and the specimens after testing are shown in Figure. 2.

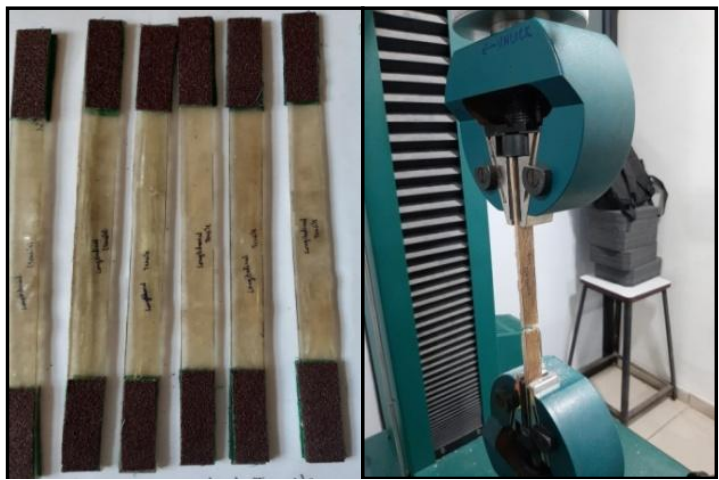

Fig. 1: Tensile testing of specimen.

B. Flexural test

The specimen for the flexural test was set up according to ASTM D-790 standard for all treated and non-treated. The 3-point flexure test was performed for set of five specimens for each sort of arrangement of fibre in composite and average estimation of flexural strength and modulus was recorded. The most normally utilized specimen size is $3.2 \mathrm{~mm} \mathrm{x}$ $13 \mathrm{~mm} \times 125 \mathrm{~mm}$. The specimen was set in UTM and force was applied until it bends thoroughly. The flexural strength and displacement were recorded. The test was conducted at room temperature. The specimens for flexural testing are shown in Figure. 3 and the specimens after testing are shown in Figure. 4.

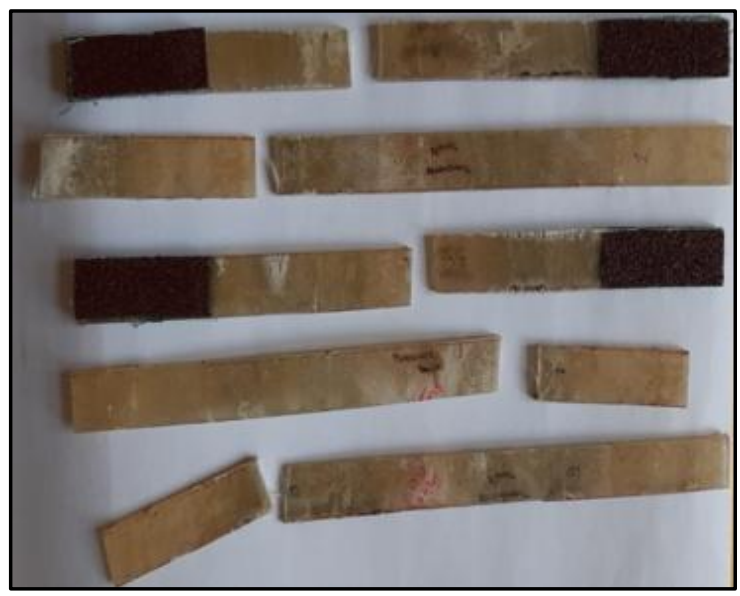

Fig. 2: Specimens after tensile test (Unidirectional continuous fibre).

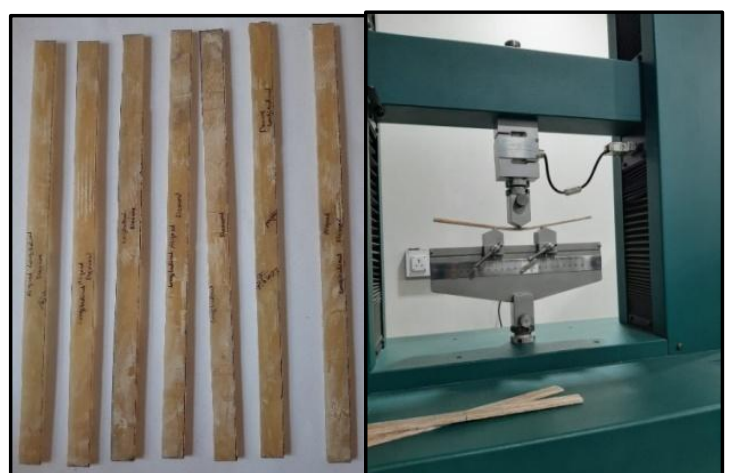

Fig. 3: Flexure testing of specimen for 3-point flexure test.

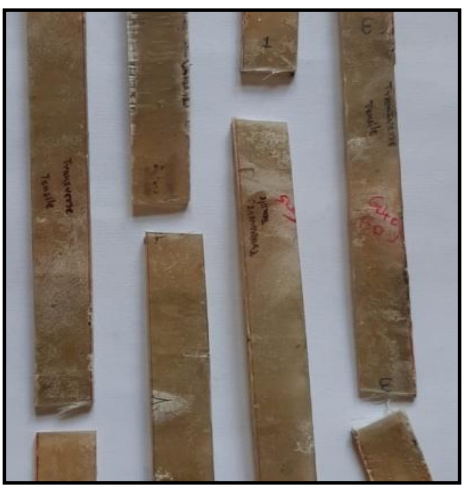

Fig.4: Specimens after flexure test (Unidirectional continuous fibre). 


\section{RESULTS AND DISCUSSIONS}

Hybrid fibre reinforced composite materials is being utilized for some, light load engineering applications throughout the previous few decades. The engineers and academicians are researching on the mechanical properties of composite for various fibre reinforcements like natural+ natural, natural + synthetic etc. In the current work, mechanical properties of hybrid fibre reinforced composite is assessed.

\section{Tensile testing}

The tensile test of composite specimen was done on the universal testing machine. The tensile load was applied till the failure of the specimens. The stress-strain values for each variety of specimens were recorded by the machine software. As per the load sustainability, different strength of the composite obtains. Tensile strength and tensile modulus of treated and untreated specimens are shown in Figure. 5 and Figure. 6 respectively.

For the tensile testing, both treated banana/glass fibre and non-treated fibre one composite get trimmed by diamond cutter in required dimension as mentioned in ASTM D3039 and testing performed on universal testing machine. Increased of reinforcement in terms of jute greater than $33.33 \%$ and $35 \%$ in comparison of glass in hybridization weaken the effect rather than strengthening as natural fibre strengthening is less than glass[8]. N. Vijay Kumar et al.[1] found tensile value for Hybrid untreated (50-50)\% glass/banana with polyester matrix with untreated and found it 120MPa. In case of chopped fibre of banana/glass, with untreated fibre reinforced composite, it is observed that as fibre length increase strength increases. Chopped fibre of $15 \mathrm{~mm}$ fibre length and $20 \%$ of fibre loading with forty percent fibre loading reported higher value as observed by R. Karthick et al[13]. Unidirectional composites are significantly proved to be stronger in the fibre direction than similar composites with fibre in multiple directions[14-17]. The high pressure and temperature in composite production allowed the discontinuous fibre to flow and distort, which led to their misalignment but in unidirectionally aligned long continuous fibre reinforced polymer composite shows more balanced mechanical performance[19-21].In the present work of unidirectional composite investigated as mentioned in Figure $5 \& 6$, it is found that treated fibre reinforced composite with volume fraction of B20-G20 has both fibre loading is of $50-50 \%$ as total of forty percent fibre loading gives maximum tensile strength of (152.3 MPa) and tensile modulus (3997 MPa) as compared to other hybrid combination. G40-B0 gives higher value than B20-G20 but due to high density, glass fibre composite observes heavy weight, so to reduce the weight natural fibre incorporation needed. In experimental investigation, it is found that the allfibre not broken at the same time, fibre of lower strength fracture first so the broken fibre transfer the load to another fibre. The first fibre to fail is basically the lower elongation fibre. The higher elongation fibre does not necessarily have a large failure strain, but it is always greater than the one of the LE fibre [18]. In this investigation, banana fibre has higher elongation as compared to lower elongation glass fibre but due to low strength it fail first so, the load then transferred to glass fibre. Hence in the stress strain diagram, it rises up to some extent and then fall. This is due to some load bearing capacity of continuous aligned glass fibre. Hence the hybrid composite with long continuous fibre observe higher strength then chopped one. Also its strength is higher as compared to nontreated because treated reduce the weight of the fibre as alkaline wash the waxy layer from the fibre surface so more banana fibre get loaded with the same weight and hence hybrid strength get enhanced.

\section{Flexure testing}

In standard ASTMD-790 taken for three point bending test of the specimen. When the composite get loaded on machine for flexure testing jaws can be adjusted on machine as per the calculation, 16t $(\mathrm{t}=$ thickness of the specimen) given in the ASTMD-790. Here the deflection of specimen observes same as that occur in the beam. On the top portion of specimen compressive stress generate while on bottom portion of loaded specimen tensile stress generated. In flexure strength (threepoint bending test) the material at the surface is strained more than the material in the bulk, when the material starts to crack. So, the 
deviation in the surface composition would strongly affect the flexural strength. Therefore, the results from the bending tests of the specimens are more sensitive to such deviation [14].

Flexure strength and Flexure modulus of treated and untreated specimens are shown in
Figure. 7 and Figure. 8 respectively. From the above graph it is found that the banana with polyester resin composite produce $41.10 \mathrm{MPa}$ of flexure strength and with treated banana fibre its strength increases to $52.3 \mathrm{MPa}$ as compared to pure polyester resin. Strength with volume fraction B10-G30 in untreated category shows maximum value.

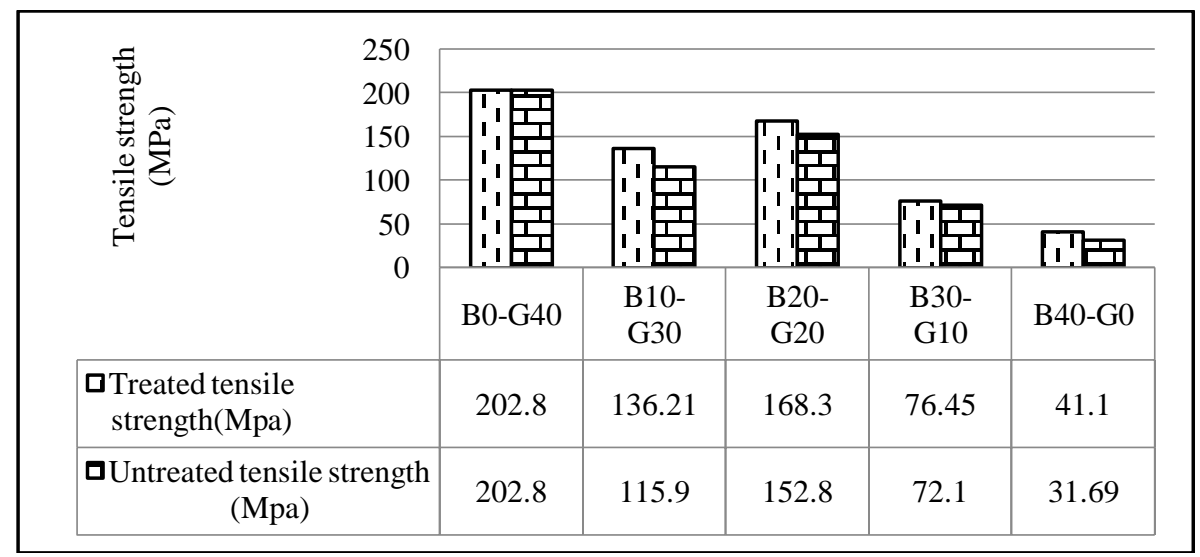

Fig. 5: Tensile strength of treated and untreated specimen.

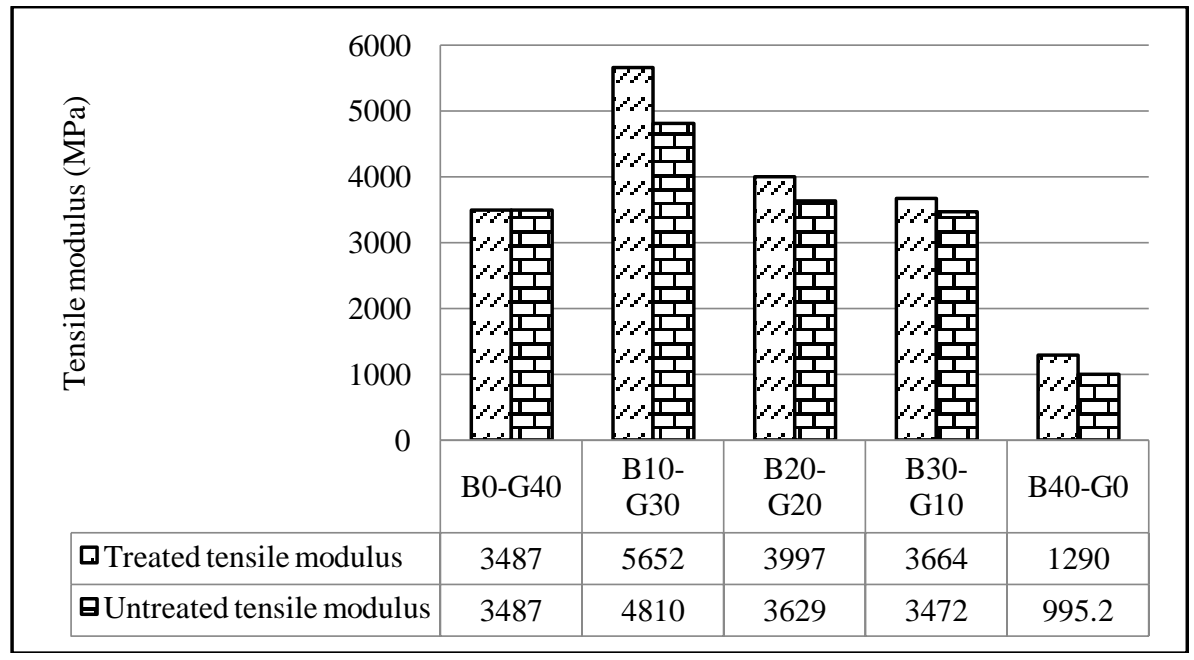

Fig. 6: Tensile modulus of treated and untreated specimen

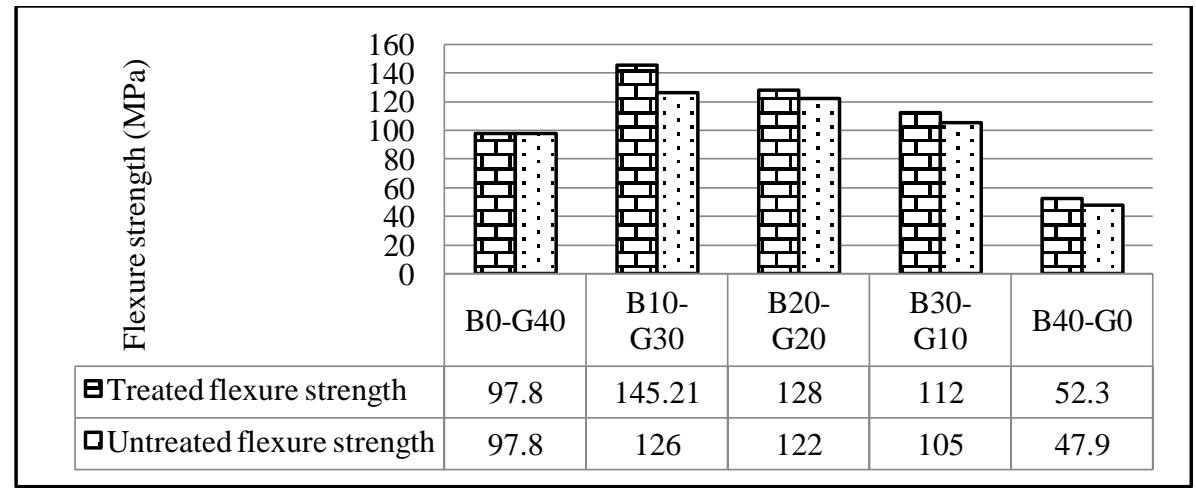

Fig. 7: Flexure strength of treated and untreated specimen. 


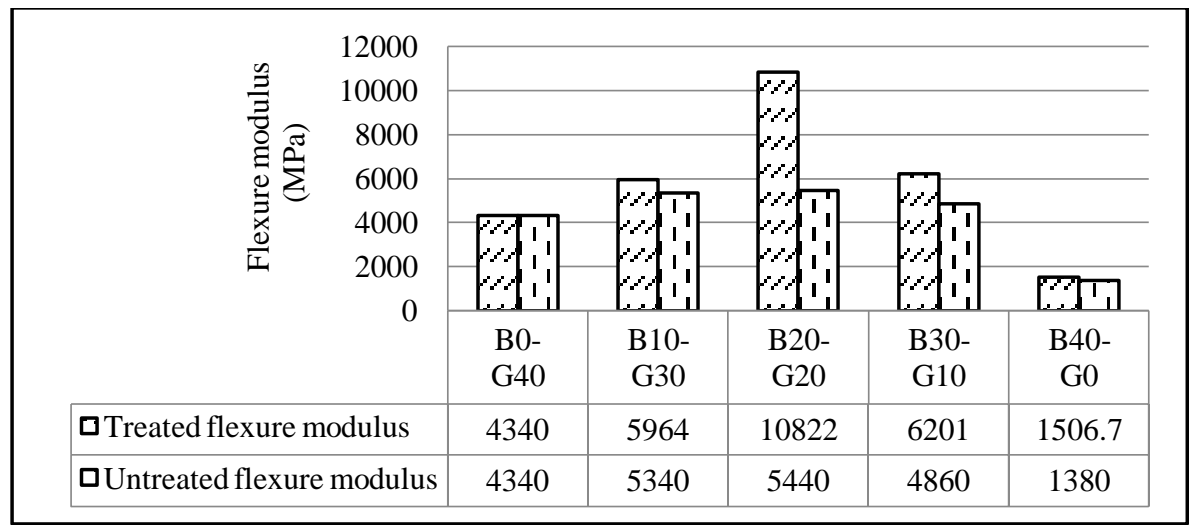

Fig. 8: Flexuremodulus of treated and untreated specimen.

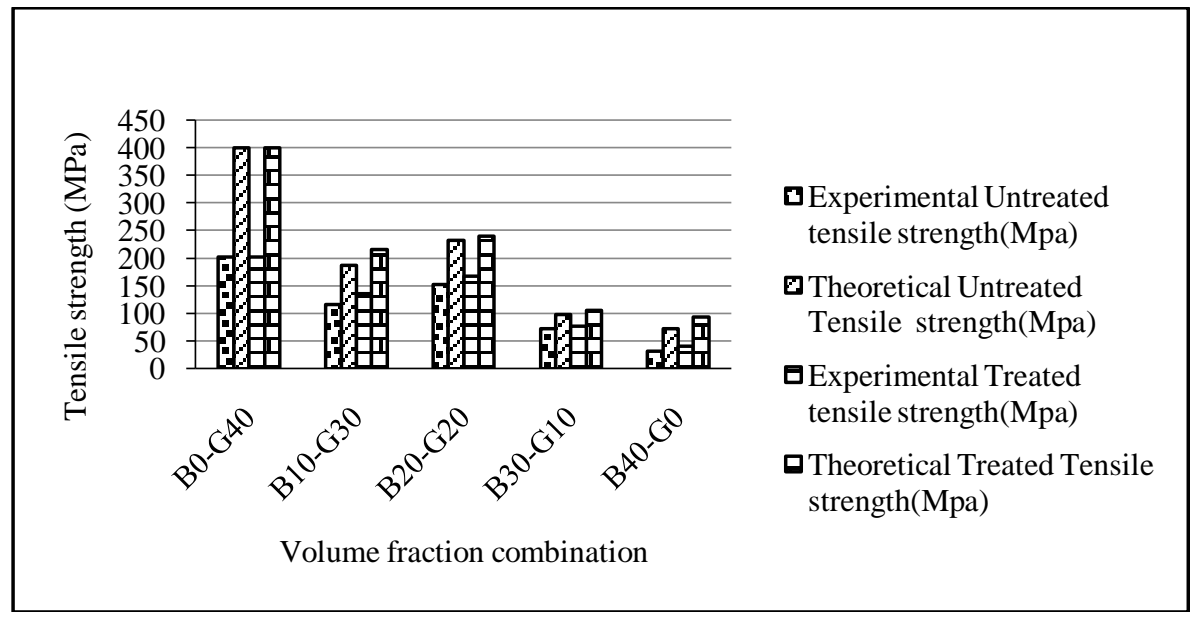

Fig. 9: Tensile strength of experimental\& theoretical.

While treated fibre of the B10-G30 gives 145.21MPa. Enhancement of the strength in treated fibre composite observes higher value in all as compared to non-treated. Maximum value of flexure strength is $145.21 \mathrm{MPa}$ and flexure modulus $5964 \mathrm{MPa}$ is obtained in this investigation.

\section{Comparisonof tensile strength}

Figure. 10 states that the tensile strength of chopped fibre composites is increased with increase in fibre length and fibre loading percentage. The maximum tensile strength was obtained in $15 \mathrm{~mm}$ fibre length and $20 \%$ fibre loading as observed by R. Karthick [13]. While in Figure. 11, it is observed that high tensile application composites may select the $25 \%$ weight fractioned treated banana fibre with the glass for the fashion of $\mathrm{G} / \mathrm{B} / \mathrm{G}$ hybrid composite. The high appreciable $45 \mathrm{MPa}$ tensile strength offers by the $25 \%$ weight fractioned treated banana fibre with the glass for the fashion of $\mathrm{G} / \mathrm{B} / \mathrm{G}$ hybrid composite. Hence the treated banana fibre improves the mechanical properties as observed by R. Saravanan [20].Figure.12 represent that as compared to mono-fibre hybrid enhance the strength and at $50 \%$ fibre loading maximum strength obtained as investigated by $\mathrm{N}$. Vijay Kumar [1].As the outcome of the present experimental work of long, continues treated fibre with unidirectional aligned fibre, great improvement in tensile strength with $\mathrm{B} / \mathrm{G}$ of $50 \%$ fibre loading is obtained as compare to $40 \%$ of B20-G20 volume fraction of fibre in the composite. The present study graph in Figure. 9 (Tensile strength of experimental \& theoretical) shows that the banana fibre with low strength but while get alkaline treated and hybridize with glass fibre increases the strength and enhance the mechanical properties. Experimental and theoretical value also observed good agreement in tensile strength. The rule of hybrid mixture value is higher than experimental value due to chance of formation of micro voids in composite. This factor was not accounted in this study. 


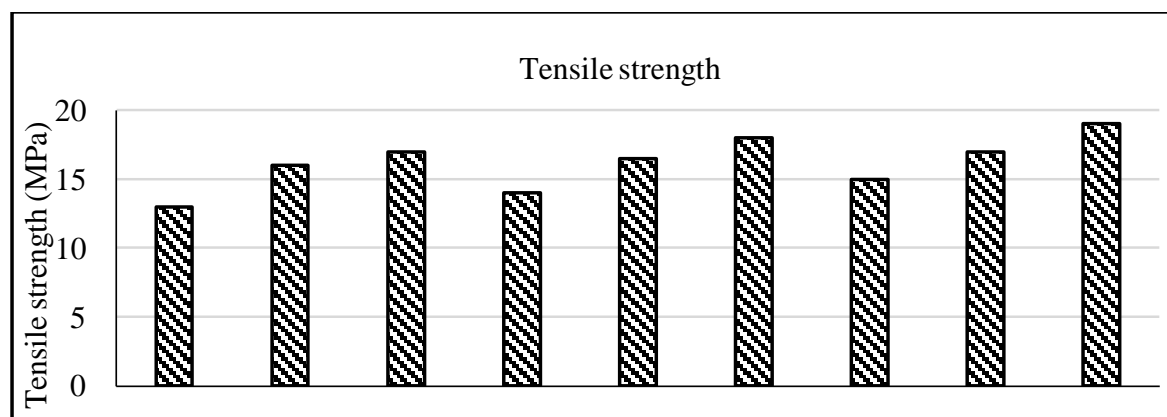

$5 \mathrm{~mm}, 10 \% 5 \mathrm{~mm}, 15 \% 5 \mathrm{~mm}, 20 \% 0 \mathrm{~mm}, 10 \% 0 \mathrm{~mm}, 15 \% 0 \mathrm{~mm}, 20 \% 5 \mathrm{~mm}, 10 \% 5 \mathrm{~mm}, 15 \% 5 \mathrm{~mm}, 20 \%$

Fibre length \& Fibre loading \%

$\mathbf{\Delta}$ Series 1

Fig. 10: Specimen tensile strength [13].

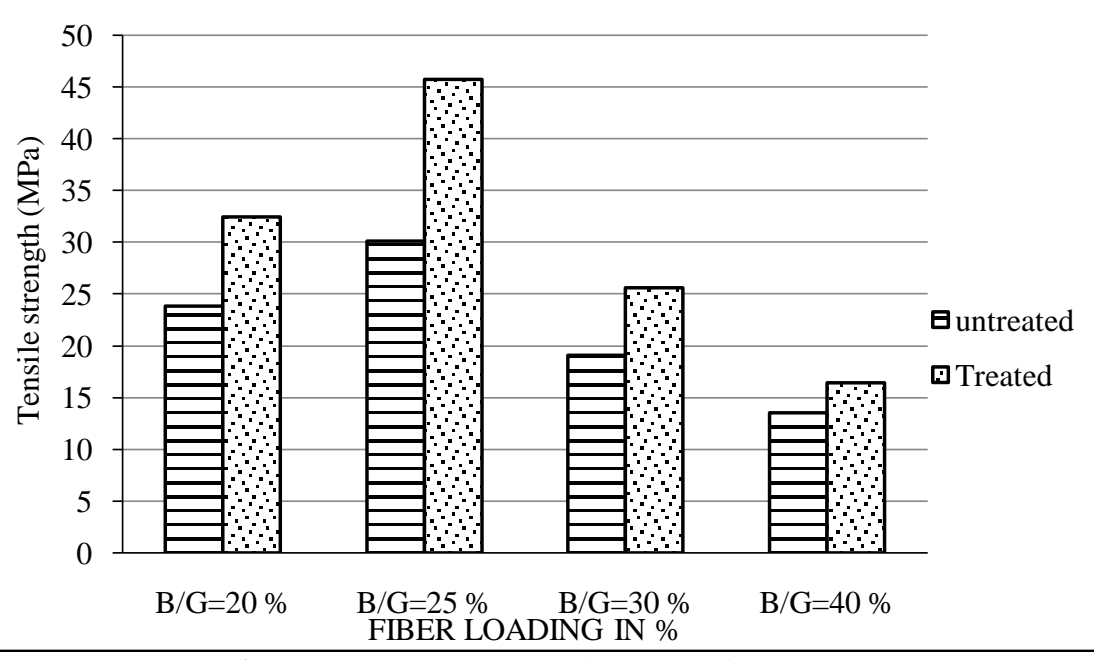

Fig. 11: Specimen tensile strength [20].

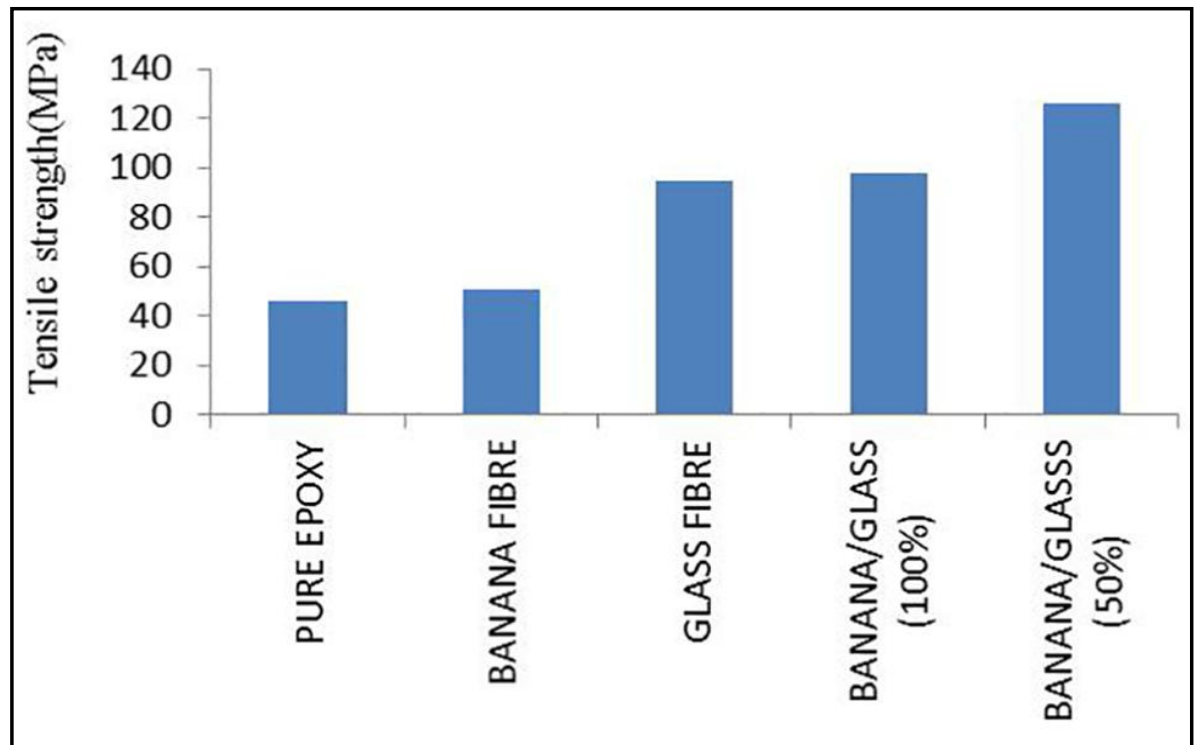

Fig. 12: Tensile strength of composites [1]. 
4. Comparison of flexure strength

Figure. 13, Figure. 14, Figure. 15, Figure. 16and Figure. 17 shows the comparison of flexural strength for different composites.

Flexural properties of untreated and treated fibre composites are shown in Figure.14. It is observed that the $100 \%$ matrix had flexural strength of $27.44 \mathrm{MPa}$ and that of untreated banana fibre reinforced composites is $36.15 \mathrm{MPa}$ with a $31.75 \%$ increase. The flexural strength of treated banana fibre composites were in the range of 50 to $73.24 \mathrm{MPa}$ with improvements in the range of $38.32 \%$ to $102.62 \%$ due to chemical treatments [22. 23]. Figure.15 Shows the relationship of volume fraction on flexural strength of specimens with treated fibre length $127 \mathrm{~mm}$ and it is concluded that with increase the fibre loading strength increase up to some extent and then decrease due to decrease the bonding of matrix and fibre. Figure 16 Shows untreated chopped fibre's flexure strength with different volume fraction and concluded that

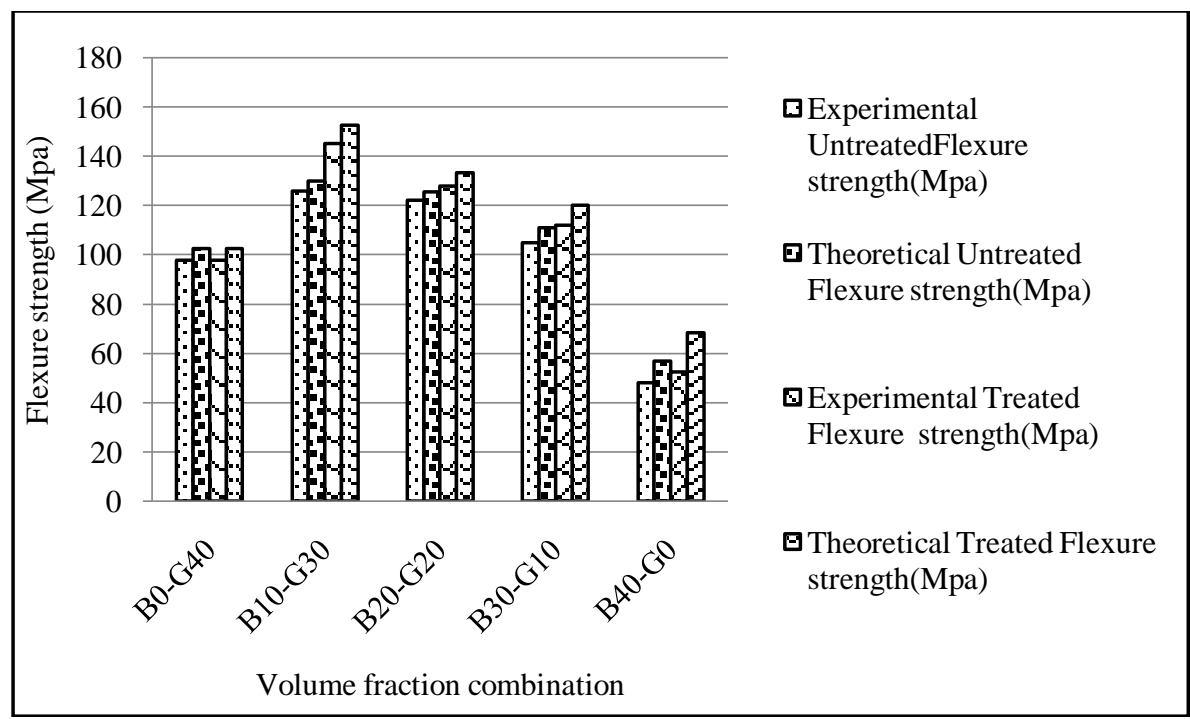

Fig. 13: Experimental v/s theoretical Flexure strength of treated and untreated.

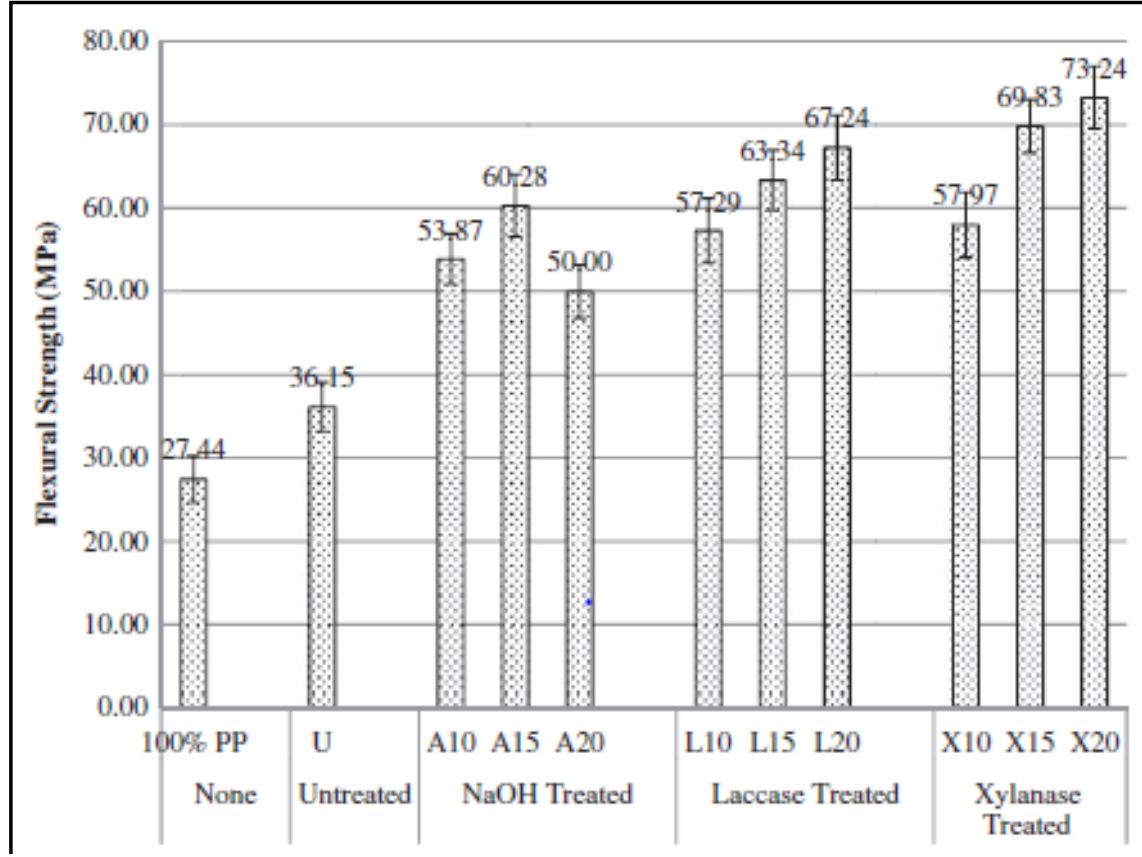

Fig. 14: Flexure strength of composites [22]. 
the $20 \%$ concentration of banana \& glass achieve higher strength as observed by V. Santhanam [2]. Figure.17 Represent untreated banana/glass fibre composite flexure strength with $20 \%$ \& $30 \%$ of total volume fraction. From the figure, it is clear that the flexural strength increases with fibre loading. At $20 \%$ fibre loading, there is a slight increase in flexural strength, i.e., 8.74 MPa. But at 30\% fibre loading, there is an increase of 53\%. The effect of fibre loading shows a similar maximum improvement with $30 \%$ fibre loading as observed by Anshida Haneefa et al.[24]. Break load of hybrid composite is high. It is about 1.26 times that of untreated composite and 1.17 times that of sisal composite [12]. So, the present study evaluate that increase of fibre loading with treated fibre will enhance the flexure strength as observed from the Figure 13 which shows that B10-G30 and B20-G20 gives maximum flexure strength with 145.21MPa and 128MPa respectively. As compared to untreated, treated reinforced composite gives enhancement of flexure strength and it is also better in theoretical comparison.

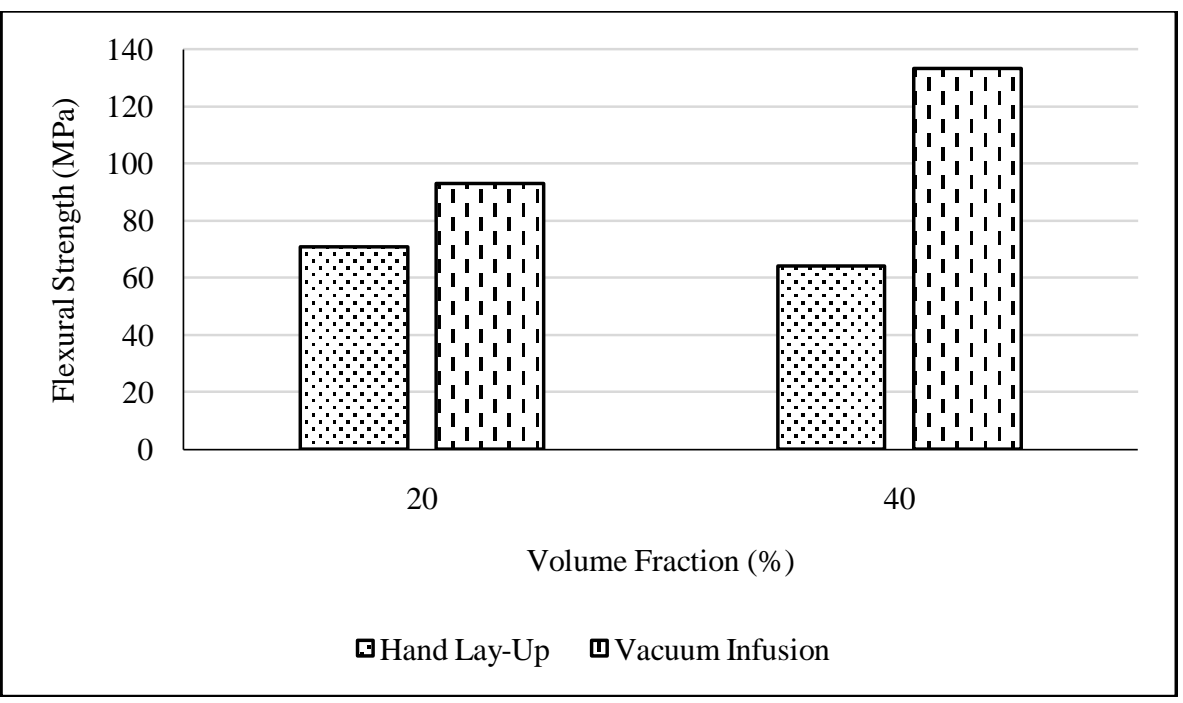

Fig. 15: Flexure strength of composites [23].

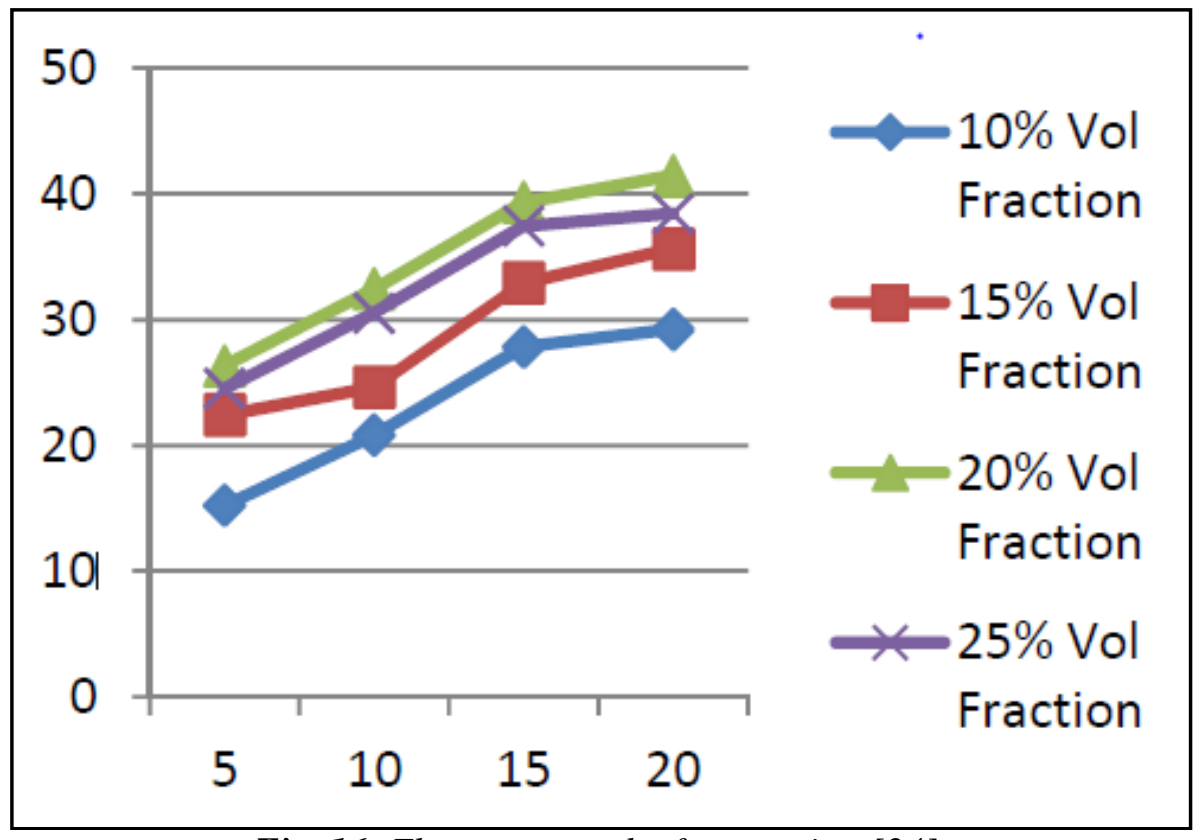

Fig. 16: Flexure strength of composites [24]. 


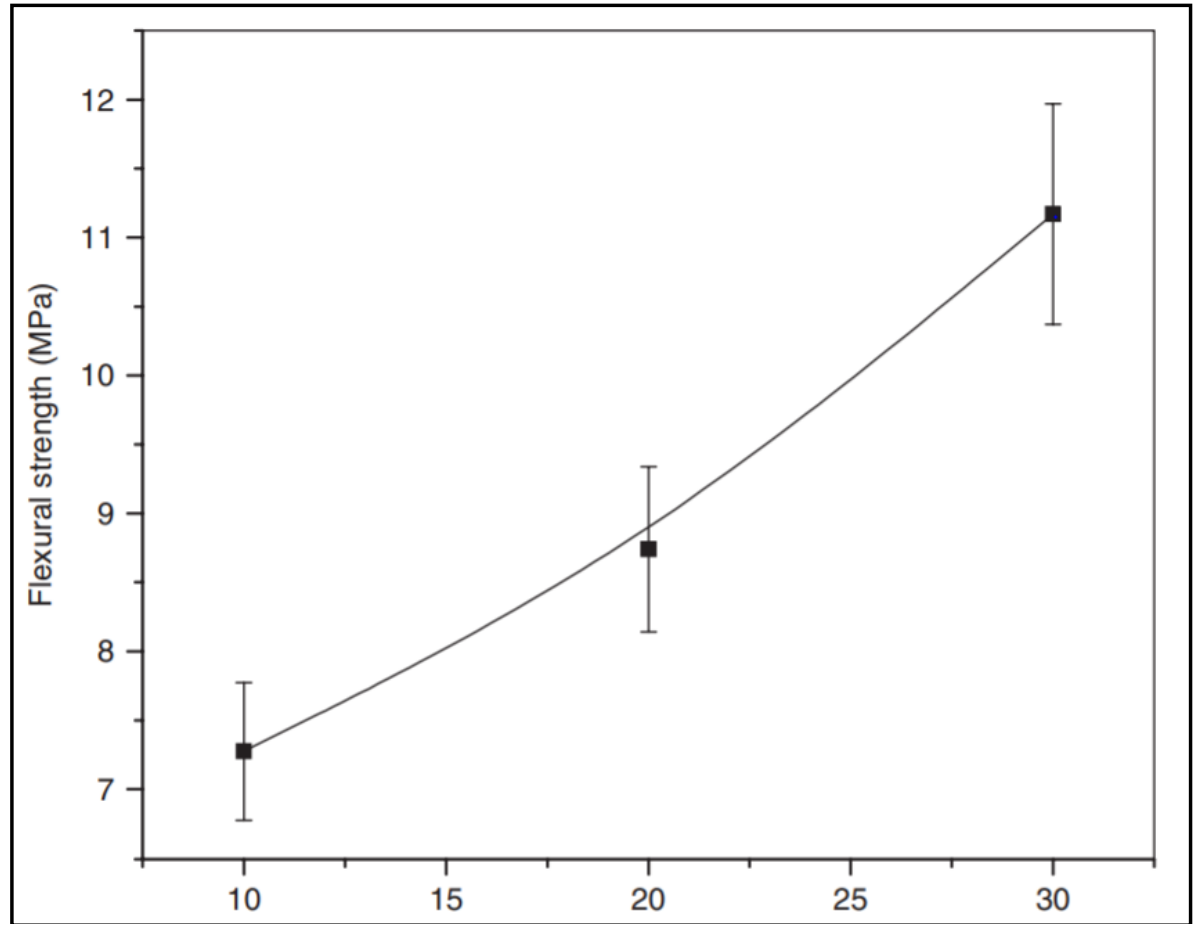

Fig. 17: Flexure strength of composites [24].

\section{CONCLUSION}

In the present study, evaluation of mechanical properties of banana/glass treated and untreated fibre reinforced composite were doneby arranging fibre in unidirectional manner.

- From the experimental investigations, it can be concluded that composite material made of $5 \% \mathrm{NaOH}$ treated bananafibre embedded with glass fibre exhibits $15.3 \%$ enhancement in the tensile strength as compared to B20-G20 of non treated value. In the same way flexure strength also increases for B10-G30 up to 10\%.

- From the comparison with the untreated fibre compositegraph, it can be concluded that hybrid effect with treated fibre increase the tensile as well as flexure strength in case of unidirectional in composite reinforcement.

- Experimental and theoretical values indicate good agreement in both tensile and flexure strength.

\section{SYMBOLS}

\begin{tabular}{l|l}
\hline$V_{c}$ & Volume of composite \\
\hline$V_{f}$ & Volume of fiber \\
\hline$V_{m}$ & Volume of matrix \\
\hline$W_{c}$ & Sum of weight of composite material \\
\hline$W_{f}$ & Weight of fibre \\
\hline
\end{tabular}

\begin{tabular}{l|l}
\hline$W_{m}$ & Weight of matrix \\
\hline$E$ & Young modulus \\
\hline$\sigma$ & Strength of composite \\
\hline$E_{m}$ & Young modulus of matrix \\
\hline$v_{f}$ & Volume fraction of fiber \\
\hline$E_{f}$ & Fibre modulus of elasticity \\
\hline$E_{m}$ & Matrix modulus of elasticity \\
\hline$F$ & Maximum load (N) \\
\hline$L$ & Distance between the support in $\mathrm{mm}$ \\
\hline$B$ & Width of the specimen in $\mathrm{mm}$ \\
\hline$H$ & Height of the specimen in $\mathrm{mm}$
\end{tabular}

\section{REFERENCES}

1. Kumar, N. V., Krishna, B. S. and Chandrika, N. S. (2019) 'Materials Today: Proceedings Evaluation of properties of glass-banana-fibre reinforced hybrid fibre polymer composite', Materials Today: Proceedings. Elsevier Ltd., 18, pp. 2137-2141. doi: 10.1016/j.matpr.2019.06.648.18.02.265.

2. Santhanam, V., Chandrasekaran, M. and Venkateshwaran, N. (2014) 'Effect of fibre parameters on the mechanical properties of Banana- Glass fibre hybrid composites', 594, pp. 202-205. doi: 10.4028/www.scientific.net/AMM.592594.202.

3. Saravanan, R. and Gnanavel, C. (2019) 'Materials Today: Proceedings Synthesis and characterization of treated banana 
fibre and selected jute fibre based hybrid composites', Materials Today: Proceedings. Elsevier Ltd, 1(xxxx), pp. 15. doi: 10.1016/j.matpr.2019.09.143.

4. Parre, A. et al. (2019) 'Materials Today: Proceedings Investigation of chemical, thermal and morphological properties of untreated and $\mathrm{NaOH}$ treated banana fibre', Materials Today: Proceedings. Elsevier

5. Jariwala, H. (2019) 'A review on mechanical behavior of natural fibre reinforced polymer composites and its applications', (June). doi: 10.1177/0731684419828524. Ltd, (xxxx). doi: 10.1016/j.matpr.2019.06.655.

6. Venkateshwaran, N., Elayaperumal, A. and Sathiya, G. K. (2012) 'Prediction of tensile properties of hybrid-natural fibre composites', Composites Part B: Engineering. Elsevier Ltd, 43(2), pp. 793796.doi:10.1016/j.compositesb.2011.08.023.

7. Enamul Hossain, M. (2011) 'The current and future trends of composite materials: An experimental study', Journal of Composite Materials, 45(20), pp. 21332144. doi: $10.1177 / 0021998311401066$.

8. Wambua, P., Ivens, J. and Verpoest, I. (2003) 'Natural fibre: Can they replace glass in fibre reinforced plastics?', Composites Science and Technology, 63(9), pp. 1259-1264. doi: 10.1016/S0266-3538(03)00096-4.

9. Komal, U. K. et al. (2018) 'ScienceDirect Effect of chemical treatment on mechanical behavior of banana fibre reinforced polymer composites'. Elsevier Ltd, 5, pp. 16983-16989. doi: 10.1016/j.matpr.2018.04.102.

10. Li, X. et al. (2007) 'Chemical Treatments of Natural Fibre for Use in Natural FibreReinforced Composites: A Review Chemical Treatments of Natural Fibre for Use in Natural Fibre-Reinforced Composites: A Review', (May 2014). doi: 10.1007/s10924-006-0042-3.

11. Pujari, S., Ramakrishna, A. and BalaramPadal, K. T. (2017) 'Prediction of Swelling Behaviour of Jute and Banana Fibre Composites by using ANN and Regression Analysis', Materials Today: Proceedings. Elsevier Ltd, 4(8), pp. 85488557. doi: 10.1016/j.matpr.2017.07.201.
12. Anand, P. (2018) 'Investigations on the performances of treated jute / Kenaf hybrid natural fibre reinforced epoxy composite'. Journal of Polymer Research.

13. Karthick, R. et al. (2018) 'ScienceDirect Evaluation of mechanical behavior of banana fibre reinforced hybrid epoxy composites', Materials Today: Proceedings. Elsevier Ltd, 5(5), pp. 12814-12820.doi:10.1016/j.matpr.20.

14. Cordin, M. and Bechtold, T. (2018) 'Physical properties of lyocell-reinforced polypropylene composites from intermingled fibre with varying fibre volume fractions'. doi: 10.1177/0892705717734594.

15. Mansor, M. R. et al. (no date) 'Stiffness Prediction of Hybrid Kenaf / Glass Fibre Reinforced Polypropylene Composites using Rule of Mixtures ( ROM ) and Rule of Hybrid Mixtures ( RoHM )', 30(3), pp. 321-334.

16. Wambua, P., Ivens, J. and Verpoest, I. (2003) 'Natural fibre: Can they replace glass in fibre reinforced plastics?', Composites Science and Technology, 63(9), pp. 1259-1264. doi: 10.1016/S0266-3538(03)00096-4.

17. Swolfs, Y., Verpoest, I. and Gorbatikh, L. (2019) 'Recent advances in fibre-hybrid composites: materials selection, opportunities and applications', International Materials Reviews. Taylor \& Francis,64(4),pp.181-215.

18. Swolfs, Y., Gorbatikh, L. and Verpoest, I. (2014) 'Fibre hybridisation in polymer composites: A review', Composites Part A: Applied Science and Manufacturing, 67, pp. 181-200. doi: 10.1016/j.compositesa.2014.08.

19. Tang, J. et al. (2019) 'Hybrid composites of aligned discontinuous carbon fibre and self-reinforced polypropylene under tensile loading, Composites Part A: Applied Science and Manufacturing. Elsevier, 123(May), pp. 97-107. doi: 10.1016/j.compositesa.2019.05.003.02

20. Saravanan, R. et al. (2019) 'ScienceDirect Synthesize and Characterization of Maleic acid Treated Banana Fibre Composites', Materials Today: Proceedings. Elsevier Ltd., 18, pp. 5382-5387. doi: 10.1016/j.matpr.2019.07.565. 
21. Swolfs, Y., Gorbatikh, L. and Verpoest, I. (2014) 'Fibre hybridisation in polymer composites: A review', Composites Part A: Applied Science and Manufacturing. ElsevierLtd,67,pp.181200.doi:10.1016/j.co mpositesa.2014.08.027.7.i:10.1080/09506 608.2018.1467365.

22. Murugan, R. and Surjit, R. (2017) 'Effect of alkali and enzymatic treatments of banana fibre on properties of banana / polypropylene composites'. doi: $10.1177 / 1528083717714479$.

23. Rahman, M. A., Jasani, A. A. and Ibrahim, M. A. (2017) 'Flexural Strength of Banana Fibre Reinforced Epoxy Composites Produced through Vacuum Infusion and Hand Lay-Up Techniques - A Comparative Study', 2, pp. 31-36.
24. O, P. H. P. (2008) 'Studies on Tensile and Flexural Properties of Short Banana / Glass Hybrid Fibre Reinforced Polystyrene Composites', 42(15). doi: $10.1177 / 0021998308092194$.

\section{Cite this Article}

Vatsal Maisuriya, Piyush Jain, Hitesh Jariwala, Damyanti Badagha. Analysis of Unidirectional Aligned Banana and Glass Fibre Reinforced Polyester Composite for Tensile and Flexural Strength. Journal of Polymer \& Composites. 2020; 8(2): 49-61p. 